\title{
Histoplasmosis: Epidemiology, Diagnosis, and Clinical Manifestations
}

\author{
Kathleen A. Linder ${ }^{1} \cdot$ Carol A. Kauffman ${ }^{1}$ \\ Published online: 7 May 2019 \\ (C) Springer Science+Business Media, LLC, part of Springer Nature 2019
}

\begin{abstract}
Purpose of Review This review highlights the epidemiology, diagnosis, and clinical manifestations of histoplasmosis. Recent Findings There is an increasing awareness of histoplasmosis in Central and South America in patients with human immunodeficiency virus/acquired immunodeficiency syndrome (HIV/AIDS), and deaths from histoplasmosis in this region may outnumber deaths from tuberculosis. Diagnosis in this region is hampered by lack of rapid diagnostic tests. Growth of H. capsulatum in culture is definitive, but takes weeks. In areas in which antigen testing is available, this has become an important rapid diagnostic tool, and methods combining antigen and antibody testing appear to improve diagnostic accuracy for acute pulmonary and central nervous system histoplasmosis. Pulmonary histoplasmosis, although usually self-limited, rarely can lead to life-threatening complications. Uncommon, but serious and sometimes fatal complications of disseminated histoplasmosis include Addison's disease, meningitis, culture-negative endocarditis, and hemophagocytic lymphohistiocytosis.

Summary The use of antigen testing has improved our ability to diagnose histoplasmosis, but these tests are not universally available. Complications of both pulmonary and disseminated histoplasmosis remain challenging.
\end{abstract}

Keywords Histoplasmosis · Histoplasma capsulatum · Antigen detection · Epidemiology · Disseminated histoplasmosis · Pulmonary histoplasmosis

\section{Introduction}

Histoplasmosis, the most common endemic fungal infection in the USA, is caused by the environmental fungus Histoplasma capsulatum. There are two variants of this fungus: Histoplasma capsulatum var. capsulatum and Histoplasma capsulatum var. duboisii. The latter has a more limited distribution, occurring primarily in Africa. This review will focus on Histoplasma capsulatum var. capsulatum (hereafter referred to as Histoplasma capsulatum).

\section{The Organism}

H. capsulatum is a member of the group of dimorphic fungi that are molds in the environment and transform into yeast-

This article is part of the Topical Collection on Clinical Pathology

Kathleen A. Linder

linderk@med.umich.edu

1 Division of Infectious Diseases, Veterans Affairs Ann Arbor Healthcare System, University of Michigan Medical School, 2215 Fuller Rd., Ann Arbor, MI 48105, USA like structures in tissues at $37^{\circ} \mathrm{C}$. The mycelial phase demonstrates $1-2.5 \mu \mathrm{m}$ septate hyphae with $2-6 \mu \mathrm{m}$ microconidia and $8-14 \mu \mathrm{m}$ macroconidia arising from lateral and terminal branches [1]. The macroconidia demonstrate finger-like protrusions called tuberculate conidia that allow tentative identification of a mold as $H$. capsulatum. The infecting conidia are the microconidia, which are of a size that can easily be inhaled into the alveoli. The yeast form is oval with a diameter of 2 $4 \mu \mathrm{m}$ that can be seen within macrophages or lying free in tissues; single budding daughter cells with a narrow base are typically noted. Atypical, enlarged, and bizarre yeast forms, along with hyphal structures, have been noted in endovascular infections $[2,3]$.

\section{The Host}

The vast majority of cases of infection with $H$. capsulatum occur in immunocompetent persons, in whom symptoms and signs are minimal. Most handle the infection quite well and do not seek medical attention [4]. However, exposure to a huge inoculum of conidia can prove to be overwhelming, even in a host with normal immune function, and can lead to severe pulmonary infection. When the person exposed to 
H. capsulatum is immunocompromised, even a small inoculum can lead to severe infection with widespread dissemination. This scenario has been noted with patients taking corticosteroids, those having received a solid organ or hematopoietic cell transplant, persons with HIV infection, and those receiving tumor necrosis factor (TNF)-alpha inhibitors [5-7].

Immunity to $H$. capsulatum is almost entirely mediated by $\mathrm{T}$ cells and activated macrophages [8]. Dissemination of Histoplasma throughout the reticuloendothelial system occurs in most patients early in the course of acute histoplasmosis, prior to the development of cellular immunity. In most cases, this is an asymptomatic event, and the host is able to control the infection when cellular immunity develops. $H$. capsulatum, similar to $M$. tuberculosis, has the ability to remain in a quiescent state for years and reactivate when cellmediated immunity is diminished by disease or treatment with immunosuppressive drugs [1].

\section{Epidemiology}

\section{Exposure Risk}

Histoplasma capsulatum is found in the soil and is more likely to be present in areas in which the soil is moist and the $\mathrm{pH}$ is close to 7.4 [9]. Soil enriched in bird and bat excrement and high in nitrogen content allows luxuriant growth of the organism. Most cases in regions in which $H$. capsulatum is commonly encountered are sporadic. Outbreaks garner more attention, especially when many hundreds or thousands of people are involved; frequently, these can be linked to a point source and activities that disperse a large number of conidia $[10-12,13 \bullet]$.

Of special importance is the risk of spelunking in caves in which large numbers of bats roost $[14,15]$. Occupational exposure, particularly among those who work in demolition or construction that involves moving soil, cleaning or sand blasting existing structures, and tearing down old structures, are at risk for exposure to large inocula of $H$. capsulatum $[13 \bullet$, $16,17]$. Travelers to the areas in which $H$. capsulatum is found are at risk for developing infection if their activities involve spelunking or disrupting soil and vegetation $[15,18,19]$.

\section{North America/Latin America}

Cases of histoplasmosis have been reported from many areas around the world, but the vast majority of cases occur in North America and Latin America [20•]. The Ohio and Mississippi River Valleys are the areas with the highest rates of infection with $H$. capsulatum, as shown by skin testing with histoplasmin decades ago [21, 22]. Additionally, microfoci of $H$. capsulatum have been linked to cases in the midAtlantic coastal states, Florida, and rural New York [23, 24].
It appears that changing patterns of human land use and possibly climate change have allowed expansion of the geographic areas in which $H$. capsulatum can survive [9]. This creates the potential for infection to be acquired outside of those areas classically thought to be endemic for this organism [25]. A small number of such cases have been noted in Montana and Idaho [26]. However, it is not totally clear that exposure to $H$. capsulatum occurred in Montana or Idaho for these six cases, because all had confirmed prior travel to the usual areas in which the organism is considered to be endemic, and reactivation could have possibly occurred. Among 12 cases described from Connecticut, 11 had lived or traveled to highly endemic areas prior to the development of infection, and 7 were immunosuppressed and at risk for reactivation of H. capsulatum [27].

In Latin America, rates of histoplasmosis have increased with increasing rates of HIV infection [28-30]. Early studies of histoplasmin reactivity outside the USA demonstrated particularly high rates of sensitivity in southern Mexico, Central America, and parts of South America [31]. Recent data suggest that more than a third of persons in Central and South America have been exposed to Histoplasma, with highest rates in Central America and northern South America (Venezuela, French Guiana, Guyana, Suriname) [29, 32]. Deaths from disseminated histoplasmosis are suspected to outnumber deaths from tuberculosis, and difficulties with rapid diagnosis of histoplasmosis in this region contribute to high mortality $[33 \bullet \bullet$.

\section{Infections Outside of the Americas}

In Africa, $H$. capsulatum occurs in microfoci, including caves, in areas that include South Africa, East Africa, and West Africa [34, 35]. The true incidence of illness due to H. capsulatum in Africa is difficult to determine because diagnostic testing is not available in many areas [36]. Cases of histoplasmosis reported from Italy, France, Germany, Spain, and other European countries are primarily noted in persons who have traveled to or have emigrated from areas of Africa that are known to be endemic for $H$. capsulatum $[37,38]$. However, autochthonous cases also are described, primarily from Italy [37, 39].

HIV infection has contributed to increased recognition of histoplasmosis in the Asia-Pacific region even though $H$. capsulatum had been isolated from soil and in caves in India, Malaysia, and Australia decades ago [36, 40, 41]. Cases of histoplasmosis originating in India are uncommon, but appear to be increasingly recognized and differentiated from tuberculosis, especially in HIV-infected persons [42, 43]. Most cases of histoplasmosis in Australia have been reported from New South Wales and Queensland, but occasional cases occur in other territories [40, 41]. Histoplasmosis competes with talaromycosis (penicilliosis) as an 
opportunistic disseminated infection in HIV-infected persons in Thailand [44]. An increasing number of cases have been reported recently from China and appear to be distributed primarily along the Yangtze River [45]. It is possible that far more cases occur in China and will be described as diagnostic methods become more widely available.

\section{Diagnosis}

The gold standard for the diagnosis of histoplasmosis involves either the recovery in culture of $H$. capsulatum from a clinical specimen or histopathological demonstration of the characteristic intracellular forms of $H$. capsulatum in infected tissues [46]. Antigen detection has become increasingly important, allowing for a rapid diagnosis of probable histoplasmosis.

\section{Histopathology}

Lymph nodes, liver, bone marrow, and skin or mucous membrane lesions are all possible sites in which biopsy may reveal H. capsulatum organisms in patients with disseminated infection. Methenamine silver or periodic-acid Schiff stains are used to visualize the characteristic 2-4 $\mu \mathrm{m}$ narrow-based budding yeast cells in samples obtained by biopsy. Granulomatous inflammation, sometimes with caseation necrosis, is the predominant pattern in tissues, but this tissue response is present in many other conditions and is not specific for histoplasmosis. In patients who are severely ill with disseminated histoplasmosis, one can sometimes see yeast forms inside circulating phagocytic cells on a routine peripheral blood smear (Fig. 1).

\section{Culture}

Samples obtained for culture should be plated on Sabouraud's dextrose agar and incubated at $25{ }^{\circ} \mathrm{C}$. At this temperature, $H$. capsulatum grows as a white to light tan mold. Growth is slow, appearing sometimes as early as 2 weeks, but often only after 4 to 6 weeks. Samples for culture from patients with disseminated infection include tissue from involved organs, such as bone marrow, liver, or mucous membrane lesions. In patients with pulmonary infections, sputum or bronchoalveolar lavage (BAL) fluid can be submitted for culture. Once growth of the mold occurs, a commercially available DNA probe specific for $H$. capsulatum is used by most laboratories for rapid definitive identification, obviating the need to convert the mold phase to the yeast phase at $37^{\circ} \mathrm{C}$ [1].

Blood cultures can yield $H$. capsulatum in patients who have disseminated disease. The sensitivity of blood cultures for growth of $H$. capsulatum is higher with the lysiscentrifugation system rather than conventional blood cultures $[47 \bullet \bullet, 48,49]$.

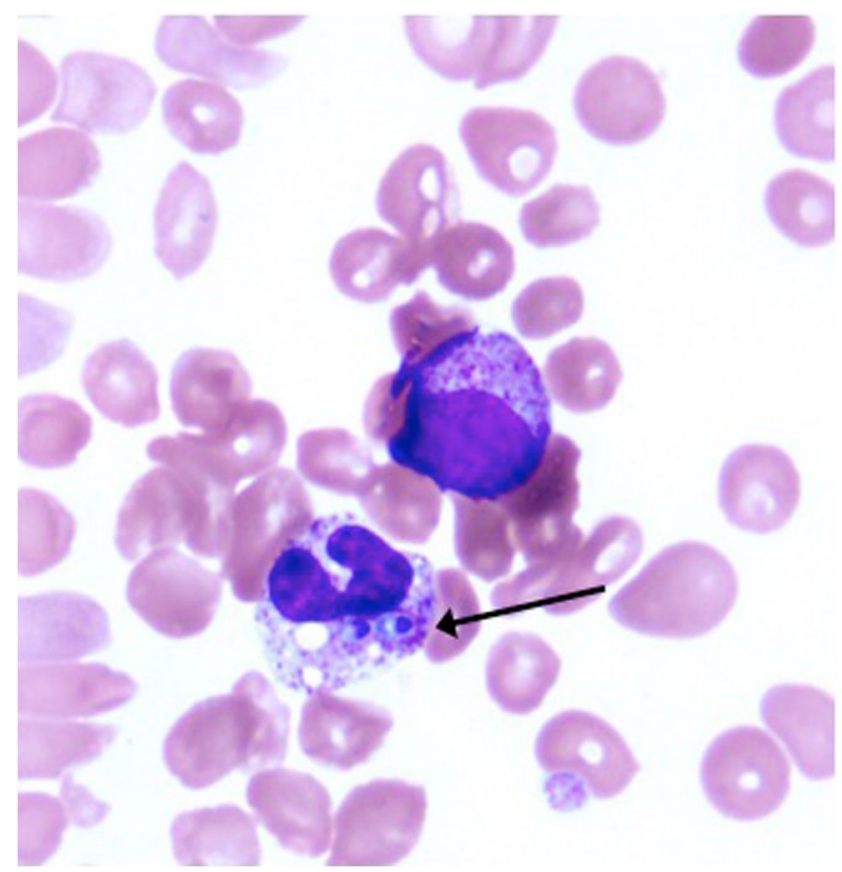

Fig. 1 Blood smear stained with Wright's stain of a patient with disseminated histoplasmosis showing a neutrophil containing two yeastlike structures typical of $H$. capsulatum (arrow)

\section{Antigen Detection}

The detection of Histoplasma galactomannan antigens has become an important testing modality for the diagnosis of histoplasmosis. First developed in 1986 as a radioimmunoassay, within a few years, it was changed to an enzyme immunoassay (EIA), which was subsequently modified several times to give a quantitative assay that can be used on serum, urine, and body fluids, such as cerebrospinal fluid (CSF) and BAL fluid $[47 \bullet \bullet, 50-52,53 \bullet]$. Most reported studies from the USA assessing sensitivity and specificity of the EIA Histoplasma antigen test have used the MiraVista assay. Sensitivity is as high as $90 \%$ in patients who have disseminated histoplasmosis, especially in those who have HIV infection and have a large burden of organisms $[54,55]$. Sensitivity is closer to $60 \%$ for patients who have acute pulmonary histoplasmosis [56].

Specificity of the MiraVista antigen assay is high when patients who have non-fungal infections are compared with those who have histoplasmosis [54]. However, the galactomannans in the cell wall of $H$. capsulatum are similar to those in the cell wall of Blastomyces dermatitidis and several other endemic mycoses (Paracoccidioides brasiliensis, Talaromyces marneffei, Coccidioides species); crossreactions are common with all of these fungi, but especially with $B$. dermatitidis. Cross-reaction with the Platelia Aspergillus galactomannan assay in patients with severe disseminated histoplasmosis is well described $[57,58]$. One drawback of the MiraVista EIA is that it is not commercially 
available and samples have to be sent to MiraVista Laboratories in Indianapolis.

Other antigen assays using EIA techniques that utilize different polyclonal or monoclonal antibodies have been developed, and those produced by ImmunoMycologics (IMMY, Norman, OK) are commercially available [59-62]. Several studies have compared either the polyclonal IMMY assay or the monoclonal IMMY assay with the MiraVista assay or with an EIA developed at the Centers for Disease Control and Prevention [60-63]. Results are mixed, with some studies noting that the IMMY tests were less sensitive than the MiraVista test $[61,63]$ but others noting very similar results among the different assays [60, 62].

\section{Antibody Testing}

Antibodies to $H$. capsulatum are generally detectable 48 weeks after initial infection in acute pulmonary histoplasmosis. Thus, testing at the time of presentation might prove negative, but follow-up antibody testing several weeks later is helpful in the diagnosis of acute infection. Patients who have disseminated histoplasmosis almost always have antibodies against $H$. capsulatum detected at the time of presentation [1]. The exception is the patient who is immunosuppressed and unable to generate a response to $H$. capsulatum antigens.

Serological tests for the diagnosis of histoplasmosis include immunodiffusion (ID), complement fixation (CF), and EIA. In the ID assay, antibodies against Histoplasma $\mathrm{H}$ and $\mathrm{M}$ antigens precipitate on an agar gel. The $\mathrm{H}$ band is seen in < $20 \%$ of cases, is rarely seen without the M band, and confirms acute infection. The $\mathrm{M}$ band is much more common and often persists after the infection has resolved [1, 47]. ID tests are approximately $80 \%$ sensitive for the diagnosis of histoplasmosis, but are more specific than CF tests [1].

$\mathrm{CF}$ testing detects the presence of antibodies against two different Histoplasma antigens, yeast phase and mycelial phase (histoplasmin) $[1,47 \bullet \bullet]$. A $\geq 4$-fold rise in antibody titer from the acute to the convalescent phase is considered diagnostic of infection with $H$. capsulatum; a single high CF titer $>1: 32$ is highly suspicious for the diagnosis of histoplasmosis. The CF assay is more sensitive than the ID assay, but crossreactions can occur with other granulomatous diseases, such as tuberculosis and sarcoidosis [1].

An EIA antibody test that can measure IgG and IgM antibodies has become available more recently $[47 \bullet \bullet, 64]$. Reports on the use of this assay have focused on acute pulmonary histoplasmosis, but this assay has also been shown to enhance the diagnosis of Histoplasma meningitis [53•]. The EIA test appears to be more sensitive than either ID or CF methods, but more experience is required in comparing this newer assay with the more standard serological techniques.

Recent studies have reported that combining antigen detection with EIA for antibodies improves the diagnosis of histoplasmosis [53•, 64]. The combination of antigen and antibody detection has been evaluated in particular for acute pulmonary histoplasmosis, in which the combined method was able to detect $96 \%$ of cases [64]. For Histoplasma meningitis, which has been notoriously difficult to diagnose, using both antigen and EIA antibody testing on CSF increased the sensitivity to $98 \%$ [53•].

\section{Molecular Tests}

For over a decade, investigators have attempted to develop a polymerase chain reaction (PCR) method that is sufficiently sensitive to aid in the diagnosis of histoplasmosis, as reviewed by Azar and Hage [47••]. Both real-time and nested PCR techniques using different molecular targets on blood or tissue samples have been used; some techniques have shown enhanced sensitivity, but at this point, there are no PCR assays readily available to the clinician to aid in the diagnosis of histoplasmosis.

\section{Clinical Manifestations}

The majority of patients who are infected with histoplasmosis have minimal signs and symptoms of infection [1]. Most infections with $H$. capsulatum are self-limited mild pulmonary infections that rarely come to the attention of physicians.

\section{Acute Pulmonary Histoplasmosis}

Symptomatic acute pulmonary histoplasmosis often presents in children or young adults upon first exposure to the organisms in the environment. It is not known what proportion of community-acquired pneumonia cases are due to histoplasmosis, but in the highly endemic areas, it is likely that this is more common than generally acknowledged. Often, the diagnosis of a fungal pneumonia is not considered until several patients present with similar symptoms within a defined time period. Then, the possibility of an environmental source for pneumonia, most likely fungal, is raised.

Symptoms suggesting acute pulmonary histoplasmosis include fever, malaise, dry cough, and substernal chest pain. Chest radiographs typically show focal airspace disease. Hilar and/or mediastinal lymphadenopathy is often present and is a clue to the diagnosis of histoplasmosis [1, 65] (Fig. 2). More severe disease occurs in cases in which exposure to a point source leads to inhalation of a large inoculum of microconidia [65]. Patients in whom this has occurred present with severe dyspnea and can quickly become hypoxic and develop acute respiratory distress syndrome (Fig. 3). 


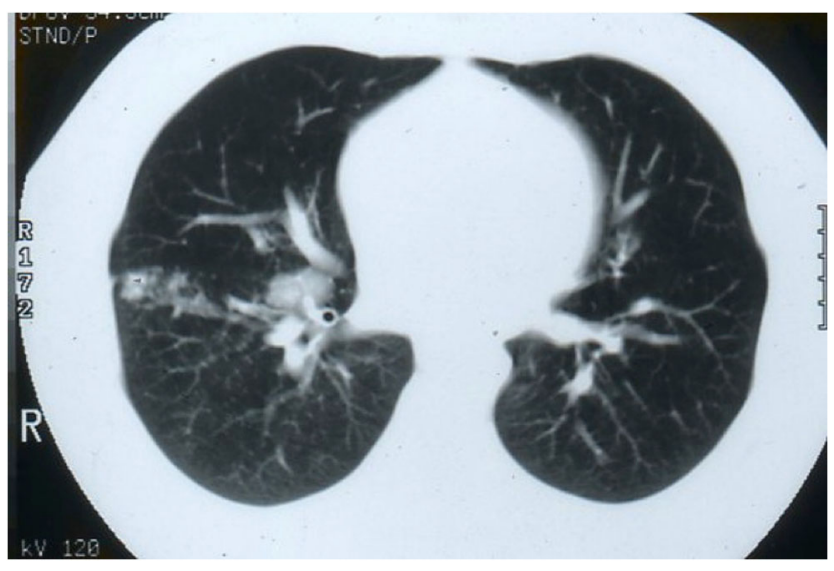

Fig. 2 CT of the thorax in a young adult who developed acute pulmonary histoplasmosis showing a localized infiltrate on the right and associated hilar lymphadenopathy

\section{Chronic Cavitary Pulmonary Histoplasmosis}

This manifestation of histoplasmosis occurs primarily in older patients with preexisting pulmonary disease. Chronic pulmonary histoplasmosis is postulated to develop as inflammation occurs adjacent to preexisting bullae, the walls of the bullae thicken, and central necrosis subsequently occurs leading to the formation of large cavitary lesions [66] (Fig. 4). In some patients, this manifests as "marching" cavities that continue to increase in size over time.

The symptoms of chronic cavitary pulmonary histoplasmosis mimic the symptoms of reactivation tuberculosis; cough, fever, and weight loss are common early in the disease course, and the cough becomes more pronounced later in the disease course. Hemoptysis occurs in about one-third of patients with significant cavitary disease [66]. A recent study found that the

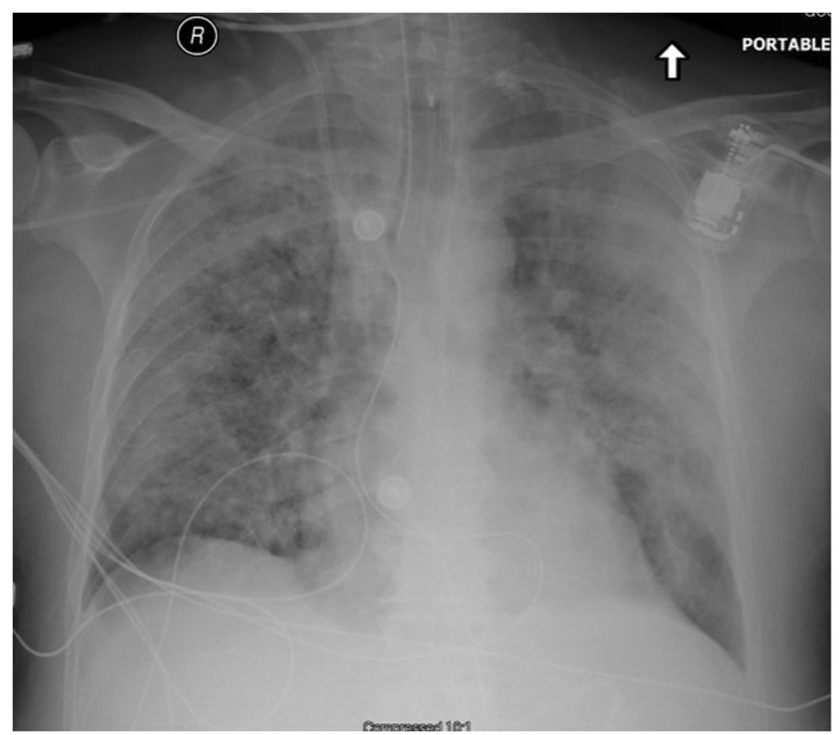

Fig. $3 \mathrm{CT}$ of the thorax in a patient who developed severe pulmonary histoplasmosis while tearing down an old barn

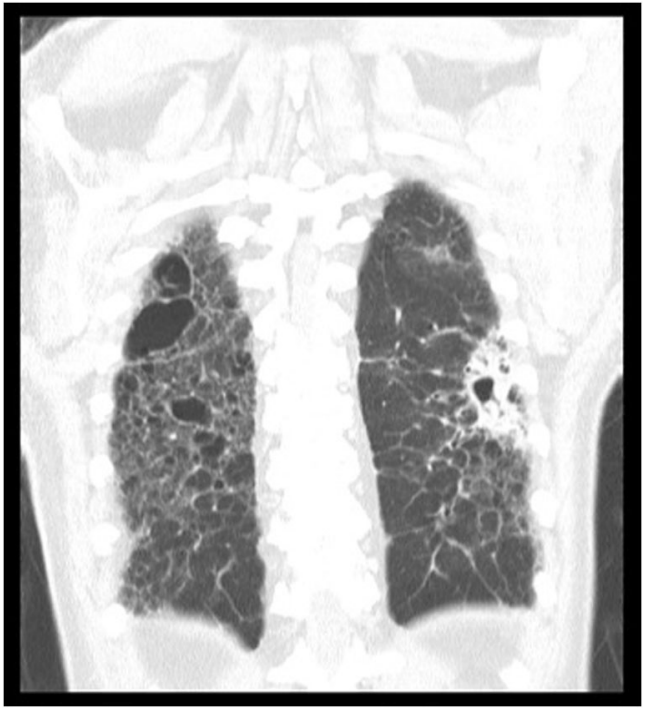

Fig. $4 \mathrm{CT}$ of the thorax in a patient with chronic cavitary pulmonary histoplasmosis. Note the extensive changes of emphysema in both lungs and the thick-walled cavity on the left

incidence of cavitation in chronic pulmonary histoplasmosis was only $39 \%$, much lower than previously noted [67]. The authors postulated that this could be explained by earlier diagnosis and treatment of this form of histoplasmosis.

\section{Complications of Pulmonary Histoplasmosis}

Granulomatous mediastinitis is an uncommon complication of pulmonary histoplasmosis in which multiple mediastinal lymph nodes enlarge to form a mass that often undergoes caseation necrosis. Patients may remain asymptomatic, or they can develop symptoms related to local encroachment upon mediastinal structures $[65,68,69]$. Thus, they might present with odynophagia, cough, chest pain, and sometimes drainage of caseous material through a sinus track.

Fibrosing mediastinitis is a rare complication of pulmonary histoplasmosis that is not related to granulomatous mediastinitis [68]. This syndrome involves a chronic inappropriate and uncontrolled fibrotic response to prior infection of the mediastinal lymph nodes. Ultimately, mediastinal fibrosis results in encasement of the structures of the mediastinum, including the pulmonary arteries and veins, the superior vena cava, or the major bronchi $[68,70]$. Patients who have unilateral fibrosis do better than those who have bilateral involvement.

\section{Disseminated Histoplasmosis}

Disseminated histoplasmosis is uncommon except in very young children and persons who are immunosuppressed, especially those who have HIV infection with CD4 cells $<150$ / $\mu \mathrm{L}[5-7,71,72]$. Another manifestation of disseminated infection is chronic progressive histoplasmosis that is most 
likely to occur in an older person [71]. A discrete source for infection often is not found in patients with disseminated infection, and reactivation of a prior infection has been documented in patients who have not returned to the endemic area [1].

Patients with disseminated histoplasmosis present with fever, fatigue, anorexia, and weight loss. Immunosuppressed patients may have overwhelming infection manifested by shock, respiratory distress, hepatic and renal failure, obtundation, and coagulopathy. Physical examination demonstrates hepatosplenomegaly and diffuse lymphadenopathy in about half of patients [72]. Painful oropharyngeal ulcerations are noted in some patients. Laboratory studies often reveal pancytopenia and elevated liver enzymes, most commonly an elevated alkaline phosphatase. Markers of inflammation, including the erythrocyte sedimentation rate, C-reactive protein, and ferritin, are elevated, and hypercalcemia secondary to granulomatous inflammation has been described [73].

Disseminated histoplasmosis can involve any organ, and autopsy studies often demonstrate involvement of organs without corresponding clinical symptoms [1, 71]. For example, gastrointestinal tract involvement is often asymptomatic or minimally symptomatic, but ulcerations, polypoid lesions, and perforations, mostly in the colon, are found in as many as $70 \%$ of patients at autopsy [71, 74].

\section{Uncommon Complications of Disseminated Histoplasmosis}

Involvement of several organ systems in patients who have disseminated histoplasmosis is uncommon, but potentially fatal if not recognized and treated promptly.

Adrenal insufficiency is a potentially life-threatening complication of disseminated histoplasmosis. In Goodwin's series of 84 patients with disseminated histoplasmosis, $82 \%$ had adrenal involvement at autopsy but only 7\% had clinically evident Addison's disease [71]. The reason that adrenal insufficiency is uncommon is most likely because both adrenal glands must be replaced with necrotizing granulomatous inflammation for insufficiency to be manifested $[71,75,76]$. Adrenal insufficiency should be considered in patients who have orthostatic hypotension, hyperkalemia, hyponatremia, and eosinophilia. Treatment of histoplasmosis does not lead to restored adrenal function, and patients continue to require corticosteroid supplementation [77].

H. capsulatum rarely causes endovascular infection, but must be considered in patients who have culture-negative endocarditis, especially when a cardiac prosthesis is involved [3]. Blood cultures yield the organism in fewer than half of the patients who have documented endocarditis; Histoplasma antigen testing has become an important part of the workup for culture-negative endocarditis in endemic regions [3]. A peculiarity unique to endovascular infection with $H$. capsulatum is that the organism can manifest both yeast and hyphal forms in the involved tissues (Fig. 5); this does not occur in other involved organs in disseminated histoplasmosis.

Meningitis occurs in $<10 \%$ of patients who have disseminated histoplasmosis and is associated with high morbidity and mortality. In immunocompromised patients, meningitis often is one aspect of widespread disseminated infection, whereas immunocompetent patients more often present with isolated chronic meningitis [78]. Common symptoms include headache, confusion, altered mental status, and focal neurological deficits. Diagnosis is often delayed, particularly in those who have no underlying immunodeficiency and have chronic meningitis. The time to diagnosis was $>26$ weeks in half of the 22 immunocompetent patients described in one series [79••].

Cultures of CSF reveal growth of $H$. capsulatum in only $25-35 \%$ of patients with meningitis, even when large volumes are cultured $[78,79 \cdot \bullet]$. Antigen testing has become the most commonly used method of making a diagnosis, but in some patients, the diagnosis is made only by finding antibody in the CSF [78]. Testing CSF for both Histoplasma antigen and antibody against Histoplasma increases the sensitivity, and it appears that the EIA antibody assay may be more sensitive in this circumstance than $\mathrm{CF}$ and ID antibodies [53・, 79••].

Hemophagocytic lymphohistiocytosis (HLH) has been reported in association with disseminated histoplasmosis, particularly in patients with AIDS who have profoundly suppressed CD4 counts [80, 81]. This life-threatening complication has been reported to resolve in some patients treated with antifungal therapy alone and without the need for further cytotoxic chemotherapy [80, 81].

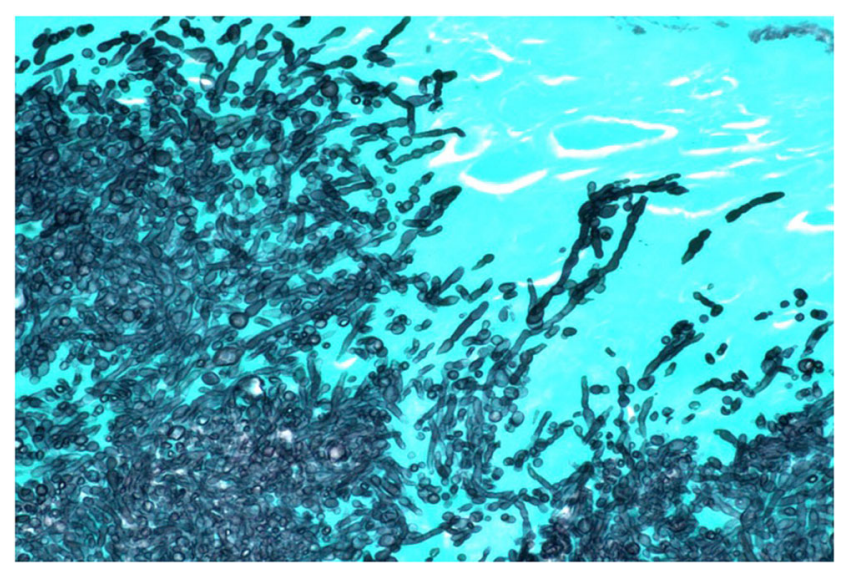

Fig. 5 Methenamine silver stain of a vegetation on the aortic valve of a patient with culture-confirmed Histoplasma endocarditis. Both hyphal forms and yeast-like forms of various sizes are seen 


\section{Conclusions}

Histoplasmosis is the most common endemic fungal infection in North and Latin America, but has been reported in small numbers from many geographic areas throughout the world. Histoplasmosis should be considered in the differential diagnosis of patients who live outside the usual endemic regions if they had previously traveled to or resided in areas known to be endemic for histoplasmosis. Exposure to soil rich in bird guano and caves (spelunking) are important clues for the diagnosis of histoplasmosis. Histoplasmosis, especially disseminated disease, has become an increasing problem in Latin America, particularly in the setting of advanced HIV/AIDS. Culture-based diagnostic methods are available in this region, but the sensitivity of culture is low and growth usually takes $4-6$ weeks. Rapid antigen testing, which has become an important diagnostic modality in the USA, is unavailable in many resource-limited settings. Complications of disseminated histoplasmosis, including adrenal insufficiency, endovascular infection, meningitis, and hemophagocytic lymphohistiocytosis, are uncommon but challenging to manage.

\section{Compliance with Ethical Standards}

Conflict of Interest The authors declare that they have no conflicts of interest.

Human and Animal Rights and Informed Consent This article does not contain any studies with human or animal subjects performed by any of the authors.

\section{References}

Papers of particular interest, published recently, have been highlighted as:

- Of importance

•. Of major importance

1. Kauffman CA. Histoplasmosis: a clinical and laboratory update. Clin Microbiol Rev. 2007;20:115-32.

2. Ledtke C, Rehm SJ, Fraser TG, Shrestha NK, Carmela DT, Rodriguez ER, et al. Endovascular infections caused by Histoplasma capsulatum. Arch Pathol Lab Med. 2012;136:640-5.

3. Riddell J, Kauffman CA, Smith JA, Assi M, Blue S, Buitrago MI, et al. Histoplasma capsulatum endocarditis: multi-center case series with review of current diagnostic techniques and treatment. Medicine (Baltimore). 2014;93:186-93.

4. Goodwin RA, Loyd JE, Des Prez RM. Histoplasmosis in normal hosts. Medicine (Baltimore). 1981;60:231-66.

5. Kauffman CA, Freifeld AG, Andes DR, Baddley JW, Herwaldt L, Walker RC, et al. Endemic fungal infections in solid organ and hematopoietic cell transplant recipients enrolled in the transplantassociated infection surveillance network (TRANSNET). Transpl Infect Dis. 2014;16:213-24.
6. Couppie P, Sobesky M, Aznar C, Bichat S, Clyti E, Bissuel F, et al. Histoplasmosis and acquired immunodeficiency syndrome: a study of prognostic factors. Clin Infect Dis. 2004;38:134-8.

7. Wood KL, Hage CA, Knox KS, Kleiman MB, Sannuti A, Day RB, et al. Histoplasmosis after treatment with anti-TNF-(alpha) therapy. Am J Respir Crit Care Med. 2003;167:1279-82.

8. Deepe GS Jr. Protective immunity in murine histoplasmosis: functional comparison of adoptively transferred T-cell clones and splenic T cells. Infect Immun. 1988;56:2350-5.

9. Maiga AW, Deppen S, Scaffidi BK, Baddley J, Aldrich MC, Dittus RS, et al. Mapping Histoplasma capsulatum exposure, United States. Emerg Infect Dis. 2018;24:1835-9.

10. Sathpatayavongs B, Batteiger BE, Wheat LJ, Slama TG, Wass JL. Clinical and laboratory features of disseminated histoplasmosis during two large urban outbreaks. Medicine (Baltimore). 1983;62: 263-70.

11. Armstrong PA, Jackson BR, Haselow D, Fields V, Ireland M, Austin C, et al. Multistate epidemiology of histoplasmosis, United States, 2011-2014. Emerg Infect Dis. 2018;24:425-31.

12. Cano MVC, Hajjeh RA. The epidemiology of histoplasmosis: a review. Semin Respir Infect. 2001;16:109-18.

13. Benedict K, Mody RK. Epidemiology of histoplasmosis outbreaks, United States, 1938-2013. Emerg Infect Dis. 2016;22:370-8 Nice review from the CDC of histoplasmosis outbreaks in the US. Notes that outbreaks are but the tip of the iceberg in terms of number of cases of histoplasmosis in the U.S. as only 10 states list histoplasmosis as a reportable infection.

14. Lottenberg R, Waldman RH, Ajello L, Hoff GL, Bigler W, Zellner SR. Pulmonary histoplasmosis associated with exploration of a bat cave. Am J Epidemiol. 1979;110:156-61.

15. Weinberg M, Weeks J, Lance-Parker S, Traeger M, Wiersma S, Phan Q, et al. Severe histoplasmosis in travelers to Nicaragua. Emerg Infect Dis. 2003;9:1322-6.

16. Armstrong PA, Beard JD, Bonilla L, Arboleda N, Lindsley MD, Chae S-R, et al. Outbreak of severe histoplasmosis among tunnel workers - Dominican Republic, 2015. Clin Infect Dis. 2018;66: $1550-7$.

17. Jones TF, Swinger GL, Craig AS, McNeil MM, Kaufman L, Schaffner W. Acute pulmonary histoplasmosis in bridge workers: a persistent problem. Am J Med. 1999;106:480-2.

18. Morgan J, Cano MV, Feiken DR, Phelan M, Monroy OV, Morales PK, et al. A large outbreak of histoplasmosis among American travelers associated with a hotel in Acapulco, Mexico, spring 2001. Am J Trop Med Hyg. 2003;69:663-9.

19. Centers for Disease Control and Prevention. Outbreak of histoplasmosis among travelers returning from El Salvador - Pennsylvania and Virginia 2008. MMWR. 2008;57:1349-53.

20. Scully MC, Baddley JW. Epidemiology of histoplasmosis. Curr Fungal Infect Rep. 2018;12:51-8 This paper is a thorough review of the global epidemiology of histoplasmosis.

21. Edwards LB, Acquaviva FA, Livesay VT, Cross FW, Palmer CE. An atlas of sensitivity to tuberculin, PPD-B, and histoplasmin in the United States. Am Rev Respir Dis. 1969;99(suppl).

22. Manos NE, Ferebee SH, Kershbaum WF. Geographic variation in the prevalence of histoplasmin sensitivity. Dis Chest. 1956;29:649 68 .

23. Morse DL, Gordon MA, Matte T, Eadie G. An outbreakof histoplasmosis in a prison. Am J Epidemiol. 1985;122:253-61.

24. Bellman B, Berman B, Sasken H, Kirsner RS. Cutaneous disseminated histolasmosis in AIDS patients in South Florida. Int J Dermatol. 1997;36:599-603.

25. Benedict K, Thompson GR, Deresinski S, Chiller T. Mycotic infections acquired outside areas of known endemicity, United States. Emerg Infect Dis. 2015;21:1935-41. 
26. Nett RJ, Skillman D, Riek L, Davis B, Blue SR, Sundberg EE, et al. Histoplasmosis in Idaho and Montana, 2012-2013. Emerg Infect Dis. 2015;21:1071-2.

27. Azar MM, Zhang X, Assi R, Hage C, What LJ, Malinis MF. +Clinical and epidemiological characterization of histoplasmosis cases in a nonendemic area, Connecticut, United States. Med Mycol 2018;56:896-899.

28. Nacher M, Adenis A, Mc DS, et al. Disseminated histoplasmosis in HIV-infected patients in South America: a neglected killer continues on its rampage. PLoS Neglected Trop Dis. 2013;7:e2319.

29. Lopes Colombo A, Tobon A, Restrepo A, Quiroz-Telles F, Nucci M. Epidemiology of endemic systemic fungal infections in Latin America. Med Mycol. 2011;49:785-98.

30. Gutierrez ME, Canton A, Sosa N, Puga E, Talavera L. Disseminated histoplasmosis in patients with AIDS in Panama: a review of 104 cases. Clin Infect Dis. 2005;40:1199-202.

31. Mochi A, Edwards PQ. Geographical distribution of histoplasmosis and histoplasmin sensitivity. Bull World Hlth Org. 1952;5:255-91.

32. Adenis A, Nacher M, Hanf M, Vantilcke V, Boukhart R, Blachet D, et al. HIV-associated histoplasmosis: early mortality and incidence trends: from neglect to priority. PLOS Neglected Trop Dis. 2014;8: e3100.

33.• Adenis AA, Valdes A, Cropet C, McCotter OZ, Derado G, Couppie $\mathrm{P}$, et al. Burden of HIV-associated histoplasmosis as compared to tuberculosis in Latin America: a modelling study. Lancet Infect Dis. 2018;18:1150-9 This paper points out the enormity of the problem of diagnosis of histoplasmosis in Latin America, emphasizing issues related to persons living with HIV infection.

34. Gugnani HC. Histoplasmosis in Africa: a review. Indian J Chest Dis Allied Sci. 2000;42:271-7.

35. Mandengue CE, Ngandjio A, Atangana PJA. Histoplasmosis in HIV-infected persons, Yaounde, Cameroon. Emerg Infect Dis. 2015;21:2094-6.

36. Bahr NC, Antinori S, Wheat LJ, Sarosi GA. Histoplasmosis infections worldwide: thinking outside of the Ohio River valley. Curr Trop Med Rep. 2015;2:70-80.

37. Ashbee HR, Evans EGV, Viviani MA, Dupont B, Chryssanthou E, Surmont I, et al. Histoplasmosis in Europe: report on an epidemiological survey from the European Confederation of Medical Mycology Working Group. Med Mycol. 2008;46:57-65.

38. Martin-Iguacel R, Kurtzhals J, Jouvion G, Nielsen SD, Libre JM. Progressive disseminated histoplasmosis in the HIV population in Europe in the HAART era. Case report and literature review. Infection. 2014;42:611-20.

39. Antinori S, Magni C, Nebuloni M, Parravicini C, Corbellino M, Sollima S, et al. Histoplasmosis among human immunodeficiency virus-infected people in Europe. Report of 4 cases and review of the literature. Medicine (Baltimore). 2006;85:22-36.

40. Chakrabarti A, Slavin MA. Endemic fungal infections in the AsiaPacific region. Med Mycol. 2011;49:337-44.

41. McLeod DSA, Mortimer RH, Perry-Keene DA, Allworth A, Woods ML, Perry-Keane J, et al. Histoplasmosis in Australia. Report of 16 cases and literature review. Medicine (Baltimore). 2011;90:61-8

42. Gopalakrishnan R, Nambi PS, Ramasubramanian V, Abdul GK, Parameswaran A. Histoplasmosis in India: truly uncommon or uncommonly recognised? J Assoc Physicians India. 2012;60:25-8.

43. Gugnani HC, Denning DW, Rahim R, Sadat A, Belal M, Mahbub MS. Burden of serious fungal infections in Bangladesh. Eur J Clin Microbiol Infect Dis. 2017;36:993-7.

44. Rangwala F, Putcharoen O, Bowonwatanuwong C, EdwardsJackson N, Kramomthing S, Kim JH, et al. Histoplasmosis and penicilliosis among HIV-infected Thai patients: a retrospective review. Southeast Asian Trop Med Public Health. 2012;43:436-41.
45. Pan B, Chen M, Pan W, Liao W. Histoplasmosis: a new endemic fungal infection in China? Review and analysis of cases. Mycoses. 2013;56:212-21.

46. De Pauw B, Walsh TJ, Donnelly JP, et al. Revised definitions of invasive fungal disease from the European Organization for Research and Treatment of Cancer/invasive fungal infections cooperative group and the National Institute of Allergy and Infectious Diseases mycoses study group (EORTC/MSG) consensus group. Clin Infect Dis. 2008;46:1813-21.

47.• Azar MM, Hage CA. Laboratory diagnostics for histoplasmosis. J Clin Microbiol. 2017;55:1612-20 Good overview of the availability and usefulness of various diagnostic tests for histoplasmosis.

48. Ramanan P, Vetter E, Milone AA, Patel R, Wengenack NL. Comparison of BACTEC MYCO/F lytic bottle to the Wampole isolator for recovery of fungal and mycobacterial organisms. Open Forum Infect Dis. 2016;3(supplement 1).

49. Vetter E, Torgerson C, Feuker A, Hughes J, Harmsen S, Schleck C, et al. Comparison of the BACTEC MYCO/F Lytic bottle to the isolator tube, BACTEC plus aerobic F/bottle, and BACTEC anaerobic lytic/10 bottle and comparison of the BACTEC plus aerobic $\mathrm{F} / \mathrm{bottle}$ to the isolator tube for recovery of bacteria, mycobacteria, and fungi from blood. J Clin Microbiol. 2001;39:4380-6.

50. Connolly PA, Durkin MM, LeMonte AM, Hackett EJ, Wheat LJ. Detection of Histoplasma antigen by a quantitative enzyme immunoassay. Clin Vaccine Immunol. 2007;14:1587-91.

51. Swartzentruber S, LeMonte A, Witt J, Fuller D, Davis T, Hage C, et al. Improved detection of Histoplasma antigenemia following dissociation of immune complexes. Clin Vaccine Immunol. 2009;16:320-2.

52. Hage CA, Davis TE, Fuller D, Egan L, Witt JR, Wheat LJ, et al. Diagnosis of histoplasmosis by antigen detection in BAL fluid. Chest. 2010;137:623-8.

53. Bloch KC, Myint T, Raymond-Guillen L, Hage CA, Davis TE, Wright PW, et al. Improvement in diagnosis of Histoplasma meningitis by combined testing for Histoplasma antigen and immunoglobulin $\mathrm{G}$ and immunoglobulin $\mathrm{M}$ anti-Histoplasma antibody in cerebrospinal fluid. Clin Infect Dis. 2018;66:89-94 This paper gives results of testing residual stored samples of CSF from patients diagnosed with Histoplasma meningitis. The authors show that sensitivity for establishing a diagnosis of Histoplasma meningitis is increased greatly when both Histoplasma antigen and antibodies against Histoplasma are sought.

54. Hage CA, Ribes JA, Wengenack NL, Baddour LM, Assi M, McKinsey DS, et al. A multicenter evaluation of tests for diagnosis of histoplasmosis. Clin Infect Dis. 2011;53:448-54.

55. Wheat LJ, Kauffman CA. Histoplasmosis. Infect Dis Clin N Am. 2003;17:1-19.

56. Swartzentruber S, Rhodes L, Kurkjian K, Zahn M, Brandt ME, Connolly P, et al. Diagnosis of acute pulmonary histoplasmosis by antigen detection. Clin Infect Dis. 2009;49:1878-82.

57. Wheat J, Wheat H, Connolly P, Kleiman M, Supparatpinyo K, Nelson K, et al. Cross-reactivity in Histoplasma capsulatum variety capsulatum antigen assays of urine samples from patients with endemic mycoses. Clin Infect Dis. 1997;24:1169-71.

58. Wheat LJ, Hackett E, Durkin M, Connolly P, Petraitiene R, Walsh $\mathrm{TJ}$, et al. Histoplasmosis-associated cross-reactivity in the BioRad platelia Aspergillus enzyme immunoassay. Clin Vaccine Immunol. 2007;14:638-40.

59. Scheel CM, Samayoa B, Herrera A, Lindsley M, Benjamin LL, Reed Y, et al. Development and evaluation of an enzyme linked immunosorbent assay to detect Histoplasma capsulatum antigenuria in immunocompromised patients. Clin Vaccine Immunol. 2009;16:852-8. 
60. Caceres DH, Samayoa BE, Medina NG, Tobon AM, Guzman BJ, Mercado D, et al. Multicenter validation of commercial antigenuria reagents to diagnose progressive disseminated histoplasmosis in people living with HIV/AIDS in two Latin American countries. J Clin Microbiol. 2018;56:1-10.

61. Theel ES, Jespersen DJ, Harring J, Mandrekar J, Binnicker MJ. Evaluation of an enzyme immunoassay for detection of Histoplasma capsulatum antigen from urine specimens. J Clin Microbiol. 2013;51:3555-9.

62. Theel ES, Harring JA, Dababneh AS, Rollins LO, Bestrom JE, Jespersen DJ. Reevaluation of commercial reagents for detection of Histoplasma capsulatum antigen in urine. J Clin Microbiol. 2015;53:1198-203.

63. Zhang C, Lei G-S, Lee C-H, Hage CA. Evaluation of two new enzyme immunoassay reagents for diagnosis of histoplasmosis in a cohort of clinically characterized patients. Med Mycol. 2015;53: 868-73.

64. Richer SM, Smedema ML, Durkin MM, Herman KM, Hage CA, Fuller D, et al. Improved diagnosis of acute pulmonary histoplasmosis by combining antigen and antibody detection. Clin Infect Dis. 2016;62:896-902.

65. Wheat LJ, Conces D, Allen SD, Blue-Hnidy D, Loyd J. Pulmonary histoplasmosis syndromes: recognition, diagnosis, and management. Semin Resp Crit Care Med. 2004;25:129-44.

66. Goodwin RA, Owens FT, Snell JD, Hubbard WW, Buchanan RD, Terry RT, et al. Chronic pulmonary histoplasmosis. Medicine. 1976;55(6):413-52.

67. Kennedy CC, Limper AH. Redefining the clinical spectrum of chronic pulmonary histoplasmosis: a retrospective case series of 46 patients. Medicine. 2007;86:252-8.

68. Parish JM, Rosenow EC, III. Mediastinal granuloma and mediastinal fibrosis. Semin Respir Crit Care Med 2002;23:135-143.

69. Hage CA, Azar MM, Bahr N, Loyd J, Wheat LJ. Histoplasmosis: up to date evidence-based approach to diagnosis and management. Sem Respir Crit Care Med. 2015;36:729-45.

70. Davis AM, Pierson RN, Loyd JE. Mediastinal fibrosis. Semin Respir Infect. 2001;16:119-30.

71. Goodwin RA, Shapiro JL, Thurman GH, Thurman SS, Des Prez RM. Disseminated histoplasmosis: clinical and pathologic correlations. Medicine (Baltimore). 1980;59:1-33.

72. Assi MA, Sandid MS, Baddour LM, Roberts GD, Walker RC. Systemic histoplasmosis: a 15 -year retrospective institutional review of 111 patients. Medicine (Baltimore). 2007;86:162-9.
73. Murray JJ, Heim CR. Hypercalcemia in disseminated histoplasmosis. Aggravation by vitamin D. Am J Med. 1985;78:881-4.

74. Lamps LW, Molina CP, West AB, Haggitt RC, Scott MA. The pathologic spectrum of gastrointestinal and hepatic histoplasmosis. Am J Clin Pathol. 2000;113:64-72.

75. Sarosi GA, Voth DW, Dahl BA, Doto IL, Tosh FE. Disseminated histoplasmosis: results of long-term follow-up. A Center for Disease Control Cooperative Mycoses Study. Ann Intern Med 1971; 75: 511-516.

76. Koene RJ, Catanese J, Sarosi GA. Adrenal hypofunction from histoplasmosis: a literature review from 1971-2012. Infection. 2013;41:757-9.

77. Singh M, Chandy DD, Bharani T, Marak RSK, Yadav S, Dabadghao P, et al. Clinical outcomes and cortical reserve in adrenal histoplasmosis- a retrospective follow-up study of 40 patients. Clin Endocrinol 2019 Jan 17 [epub ahead of print].

78. Wheat LJ, Musial CE, Jenny-Avital E. Diagnosis and management of central nervous system histoplasmosis. Clin Infect Dis. 2005;40: 844-52.

79.• Wheat J, Myint T, Guo Y, Kemmer P, Hage C, Terry C, et al. Central nervous system histoplasmosis: multicenter retrospective study on clinical features, diagnostic approach and outcome of treatment. Medicine. 2018;97:13(e0245 This is a retrospective review of 77 cases of CNS histoplasmosis and provides details about diagnostic testing and treatment.

80. Gomez-Espejo SM, Olalla-Sierra J, Mari-Jimenez P, PeredaSalguero T, Perez-Stachowski J, de-la-Torre-Lima J, et al. Reconstitution inflammatory syndrome like reactive hemophagocytic syndrome associated with disseminated histoplasmosis in a HIV patient. Mycopathologia 2017;182:767-770.

81. Castelli AA, Rosenthal DG, Bender Ignacio R, Chu HY. Hemophagocytic lymphohistiocytosis secondary to human immunodeficiency virus-associated histoplasmosis. Open Forum Infect Dis. 2015;2. https://doi.org/10.1093/ofid/ofv055.

Publisher's Note Springer Nature remains neutral with regard to jurisdictional claims in published maps and institutional affiliations. 